\title{
Comparison of Analgesic Effects of Novafen and Ibuprofen after Periodontal Surgeries
}

Amirreza Babaloo ${ }^{1}$, Mahdi Rahbar ${ }^{2}$, Shima Ghasemi ${ }^{3 *}$, Mohammadtaghi Chitsazi $^{4}$, Hamed Moradzadeh ${ }^{5}$

1. Assistant Professor, Department of Periodontics, Faculty of Dentistry, Tabriz University of Medical Sciences, Iran 2. Post-graduate Student, Department of Operative \& Esthetic Dentistry, Faculty of Dentistry, Tabriz University of Medical Science, Tabriz, Iran

3. Assistant Professor, Department of Prosthodontics, Faculty of Dentistry, Tabriz University of Medical Sciences, Iran

4. Professor, Department of Periodontics, Faculty of Dentistry, Tabriz University of Medical Sciences, Iran

5. Dentist, Private practice, Tabriz, Iran

\begin{abstract}
Introduction: Management of pain after dental procedures is one of the most important issues for dentists. Non-steroidal antiinflammatory agents, such as ibuprofen, are one of the most commonly used medications to relieve pain. Recently a new medication, Novafen, has been introduced into the Iranian pharmaceutical market, which is a combination of ibuprofen, acetaminophen and caffeine. Considering the importance of management of pain after periodontal surgeries and a paucity of studies in this respect, the present study was undertaken to compare the analgesic efficacy of Novafen and ibuprofen in alleviating pain after periodontal surgeries. Materials and methods: In the present controlled randomized clinical trial, 30 systemically healthy subjects with moderate to severe generalized chronic periodontitis, who were candidates for flap surgeries were evaluated in two groups with 30 areas in each group (two areas in each patient). After the periodontal surgical procedures, the subjects in group 1 received Novafen capsules (containing $325 \mathrm{mg}$ of acetaminophen, $200 \mathrm{mg}$ of ibuprofen and $40 \mathrm{mg}$ of caffeine) and the subjects in group 2 received ibuprofen $(400 \mathrm{mg})$. The medications were selected from one pharmaceutical company and the patients only used the medications they received. The severity of pain was determined and compared at 3minute, 1-hour and 3-hour intervals using VAS and 1, 2 and 3 days postoperatively using VRS. Results: Severity of pain at 30-minute interval in the Novafen group was significantly less than that in the ibuprofen group, with no significant differences at 1- and 3-hour intervals. However, VRS revealed significantly less pain 1,2 and 3 days postoperatively in the Novafen group compared to the ibuprofen group. Conclusion: Based on the results of the present study, it can be concluded than Novafen can be more effective after periodontal surgeries in alleviating pain. However, its pain control capacity was similar to that of ibuprofen during the early postoperative hours, with better performance 1-3 days postoperatively.
\end{abstract}

Key words: Novafen; ibuprofen; pain relief; periodontal surgery; Analgesia;

\section{Corresponding author:}

Shima Ghasemi

Department of Prosthodontics, Faculty of Dentistry, Tabriz University of Medical Sciences, Tabriz, Iran Tel: +989143180309 E-mail:dr_shimaghasemi@yahoo.com

Receive date: 2016-11-01 | Accept date: 2016-12-21 | Publish date: 2017-02-10

DOI: 10.7575/aiac.abcmed.17.05.01.01 


\section{Introduction}

Pain is a defense mechanism of the body, which is empirically distinguished from other senses such as touch and perception of heat, cold, pressure etc; pain is an internal feeling (1). Postoperative pain and discomfort and edema after dental procedures, especially after periodontal procedures, have a high prevalence rate. In particular, pain and discomfort that occur during the first 24 hours after periodontal surgeries are classified as moderate to severe pain in dentistry (2). Non-steroidal antiinflammatory drugs (NSAIDs) are a heterogeneous group of medications that usually have no chemical relationship with each other, although the majority of them are organic acid derivatives (3). The drugs predominantly exert their effects through inhibition of the synthesis of prostaglandins (4).

One of the most commonly administered NSAIDs is ibuprofen which is widely used for the relief of pain and is a simple derivative of phenylpropionic acid, with antiinflammatory and analgesic effects. Various side effects have been reported for ibuprofen, including gastrointestinal disorders, platelet aggregation defects, renal function disorders, skin eruptions, urticaria, ear whizzing, confusion, headaches, aseptic meningitis, etc (5).

Another analgesic agent is acetaminophen, which is safe even at its maximum dose which is $4 \mathrm{~g} /$ day. However, it is not very effective in relieving severe pain. In addition, it can be used as a selective analgesic in association with ibuprofen $(5,6)$.

There is also evidence that caffeine, alone or in combination with acetaminophen, ibuprofen or aspirin, can improve their analgesic effects (7). Evaluation of the efficacy of celecoxib, naproxen and ibuprofen in relieving pain after periodontal surgeries showed that the analgesic effects of ibuprofen and celecoxib was similar but higher than that of naproxen (8).

Comparison of the analgesic effects of ibuprofen and Aulin (the specific inhibitor of Cox-2) after gingival graft procedures showed that during the first 9 hours after surgery ibuprofen exhibited higher analgesic effects than Aulin. However, during the first and second post-operative days, Aulin exhibited higher analgesic effects compared to ibuprofen. However, no significant differences were detected between the two groups (9).

Novafen is a new medication which is a combination of acetaminophen, ibuprofen and caffeine and has been marketed in Iran recently (7). Only a few studies have compared the analgesic effects of ibuprofen and Novafen, including a study by Raeisian et al, who compared the analgesic effects of ibuprofen and Novafen after surgical extraction of mandibular third molars. They concluded that there were no differences between the analgesic effects of Novafen and ibuprofen after such surgeries (10). In contrast, a study by Merry et al on the effects of Novafen and ibuprofen on pain relief after extraction of third molars in adult patients showed that Novafen was much more effective than Novafen (6).

This study is the first one to compare the analgesic effects of Novafen and ibuprofen after periodontal surgeries. Data were collected and analyzed to answer the question whether the analgesic effects of Novafen and ibuprofen were different or not.

\section{Materials and Methods}

The subjects in this controlled randomized clinical trial were selected from those referring to the Department of Periodontics, Tabriz Faculty of Dentistry in 2015. Data were collected using a checklist, and Power \& Sample Size software program and similar studies were 
used to determine the sample size. A total of 27 samples were calculated for each group by condsidering $\alpha=0.05$, a power of $80 \%$ and mean pain scores of $1.3 \pm 1.2$ and $2.6 \pm 2.4$ in the two groups receiving analgesics based on previous studies (8); however, in order to improve accuracy and by considering the loss of $10-15 \%$ of the subjects during the study, 30 subjects were included in the study, adding up to 60 regions in all. All the subjects signed an informed consent form before being included in the study. No extra costs were inflicted on the subjects for the medications they received and they were all free to leave the study whenever they wished to. In addition, no names of the patients were mentioned anywhere.

The inclusion criteria consisted of signing an informed consent form, an age range of 16-40 years and affliction with moderate to severe generalized chronic periodontitis, requiring pocket elimination surgery. The exclusion criteria consisted of systemic or mental diseases, abuse of alcohol or any other psychotropic agents, any known allergy to ASAIDs, caffeine or acetaminophen, affliction with any hemorrhage disease, susceptibility to any infections after surgeries, continuous use of aspirin or ASAIDs and presence of carious teeth in the surgical field. Based on the criteria mentioned above, 30 systematically healthy subjects who had moderate to severe generalized chronic periodontitis, who were candidates for maxillary surgical flaps for pocket elimination or decreasing pocket depths (with probing pocket depths over $5 \mathrm{~mm}$ ) were selected. Each quadrant in each patient underwent a separate surgery at an interval of 14 days and the study was carried out using a split mouth design.

Each quadrant which was a candidate for periodontal surgery (the maxillary left and right quadrant) in each patent was randomly assigned to one group using the randomizer website at randomizer.org, in order to receive Novafen capsules (containing $325 \mathrm{mg}$ of acetaminophen, $200 \mathrm{mg}$ of ibuprofen and 40 $\mathrm{mg}$ of caffeine) or $400 \mathrm{mg}$ of ibuprofen after periodontal surgery. There was an interval of 14 days between the two surgeries. The medications were all selected from one pharmaceutical company. All the subjects only used the medications given. On the other hand, the patients did not take any analgesics for at least 4 hours prior to the surgery. The first dose of the analgesics was given immediately after surgery and the subsequent doses were taken at 6-hour intervals. The medications were given for 3 days. The patients' pain severity was evaluated at 30-minute, 1-hour and 3-hour postoperative intervals using VAS and at 1-, 2and 3-day postoperative intervals with the use of VRS (verbal rating scale). Evaluation of pain based on VRS was carried out using a telephone call and the pain severity was scored based on the following scoring system: 0: no pain; 1 : mild pain (tolerable); 2 : moderate pain (considerable pain that was relieved after taking the analgesic given); 3: moderate pain (considerable pain that was not relieved after taking the analgesic given); 4: severe pain. It should be pointed out that none of the patients was given any antibiotics. Finally, data were recorded in a questionnaire and analyzed with SPSS 21, using descriptive statistics (frequencies, percentages, means \pm standard deviations). T-test was used to compare quantitative variable between the two groups. Statistical significance was defined at $\mathrm{P}<0.05$.

\section{Results}

A total of 30 patients with 60 quadrants (30 quadrants in each group) were evaluated in the present study and the results are presented in Tables 1 and 2.

Based on VAS, the mean scores of pain severity of patients at 30-minute and 1- and 3hour intervals after periodontal surgeries are as follows (Table 1 ):

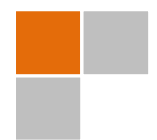


- $\quad$ At 30-minute postoperative interval, the VAS means were $2.1 \pm 0.80$ and $2.73 \pm 0.58$ in the Novafen and ibuprofen groups, respectively, with significantly lower mean in the Novafen group $(\mathrm{P}=0.001)$.

- At 1-hour postoperative interval, the VAS means were $2.59 \pm 0.97$ and $2.66 \pm 0.71$ in the Novafen and ibuprofen groups, respectively, with no significant differences between the two groups ( $P=0.547)$.

- At 3-hour postoperative interval, the VAS means were $2.33 \pm 1.26$ and $2.60 \pm 0.72$ in the Novafen and ibuprofen groups, respectively, with no significant differences between the two groups $(P=0.321)$.

In addition, the mean VRS scores of patients at 1-, 2- and 3-day postoperative intervals were as follows (Table 2):

- At 1-day postoperative interval, the VRS means were $1.63 \pm 1.06$ and $2.20 \pm 0.76$ in the Novafen and ibuprofen groups, respectively, with significantly lower mean in the Novafen group ( $\mathrm{P}=0.021)$.

- At 2-day postoperative interval, the VRS means were $1.06 \pm 0.94$ and $1.93 \pm 0.82$ in the Novafen and ibuprofen groups, respectively, with significantly lower mean in the Novafen group ( $\mathrm{P}=0.009)$.

- At 3-day postoperative interval, the VRS means were $0.53 \pm 0.68$ and $1.63 \pm 0.76$ in the Novafen and ibuprofen groups, respectively, with significantly lower mean in the Novafen group ( $\mathrm{P}=0.001)$.

\section{Discussion}

Postoperative pain is a complication that is associated with an unpleasant experience for patients (2). After surgical operations, the trauma and tissue injuries result in the disruption of cell membranes with the help of phospholipases, leading to the release of phospholipids that are in turn converted to prostaglandins, tromboxane and other metabolites by Cox-1 and Cox-2 enzymes, finally producing pain (6). One of the most commonly used drug groups that are used to prevent and relieve pain are non-steroidal antiinflammatory drugs (NSAIDs) that relieve pain by inhibiting prostaglandins $(1,12)$. Apart from NSAIDs, acetaminophen or paracetamol is another medication that has less side effect despite high efficacy in relieving pain (3). Recently, a new analgesic agent has been marketed in Iran in a capsule form, referred to as Novafen, which is a combination of ibuprofen, acetaminophen and caffeine (7).

The severity of pain depends on various factors, including the type of dental treatment (endodontic or periodontal surgery), the type of periodontal surgery (pocket elimination or crown lengthening), the patient's psychological status, patient's gender, duration of surgery, the severity of trauma inflicted, the duration of the gingival disease, etc (3). Therefore, in the present study, attempts were made to match the subjects in the two groups in relation to age, gender and the type of surgery as for as possible.

Based on the results of the present study, the severity of pain at 30-minute postoperative interval (based on VAS) and at 1-, 2- and 3-day postoperative intervals (based on VRS) was significantly lower in the Novafen group compared to the ibuprofen group, consistent with other studies in this respect. For example, Merry et al compared the use of acetaminophen- and ibuprofen-containing medications with ibuprofen alone and concluded that combined medications were more effective in relieving pain after periodontal surgeries, which is consistent with the results of the present study (6).

In another study by Rashwan et al, use of a combination of acetaminophen-caffeine after open flap debridement was more effective in relieving pain during the first hours compared to ibuprofens. In the present study, Novafen (a 


\begin{tabular}{cccc}
\hline & \multicolumn{2}{c}{ Mean score based on VAS } & \multirow{2}{*}{ P-value } \\
\cline { 2 - 3 } Postoperative interval & Ibuprofen & Novafen & \\
\hline 30 minutes & $2.73 \pm 0.58$ & $2.1 \pm 0.80$ & $* 0.001$ \\
\hline 1 hour & $2.66 \pm 0.71$ & $2.53 \pm 0.97$ & 0.547 \\
\hline 3 hours & $2.60 \pm 0.72$ & $2.33 \pm 1.26$ & 0.321
\end{tabular}

*significant statistically

Table 1: Patients' pain scores after periodontal surgeries based on VAS

\begin{tabular}{cccc}
\hline & \multicolumn{2}{c}{ Mean score based on VRS } & \multirow{2}{*}{ Postoperative interval } \\
\cline { 2 - 3 } $\mathbf{1}$ day & lbuprofen & Novafen & \\
\hline $\mathbf{2}$ days & $2.20 \pm 0.76$ & $1.63 \pm 1.06$ & $*^{*} 0.021$ \\
\hline $\mathbf{3}$ daya & $1.93 \pm 0.82$ & $1.06 \pm 0.94$ & $*^{*} 0.001$ \\
\hline & $1.63 \pm 0.76$ & $0.53 \pm 0.68$ & $* 0.001$ \\
\hline
\end{tabular}

*significant statistically

Table 2: Patients' pain scores after periodontal surgeries based on VRS

combination of acetaminophen, ibuprofen and caffeine) was significantly more effective than ibuprofen in relieving pain during the first hour after surgery; however, at 2- and 3-hour postoperative intervals, although the subjects in the Novafen group reported less severe pain, the difference was not significant, which might be attributed to the half life of ibuprofen, acetaminophen and caffeine. In this context, all these three medications begin to be metabolized after 2 hours; therefore, the difference in pain relief between the subjects taking them decreases. On the other hand, caffeine can increase gastrointestinal movements and increase absorption of other analgesics. Therefore, presence of caffeine along with other analgesics is recommended to increase their efficacy (13). Mehrvarz et al compared the analgesic effects of naproxen, Novafen and tramadol in preventing pain after radicular surgeries with those of a placebo and reported that all the three medications were effective (14).

In another study, Wan et al reported that use of ibuprofen in combination with caffeine is responsible for improving the analgesic efficacy of ibuprofen in comparison with ibuprofen alone, rather consistent with the results of the present study, which might be attributed to the analgesic effects of caffeine and its effect on increasing gastrointestinal motility (15).

Based on the results and limitations of the present study, it is suggested that similar studies be carried out in future with larger sample sizes for better generalization of the results. In addition, it is suggested that studies be carried out to evaluate the side effects of these two medications after periodontal surgeries. 


\section{Conclusion}

It can be concluded from the results of the present study that the use of a combination of acetaminophen, ibuprofen and caffeine (referred to as Novafen) can result in better analgesic effects after periodontal surgeries. In fact, the analgesic effects of Novafen were significantly higher than those of ibuprofen at 30-minute and 1-, 2- and 3-day intervals postoperatively. In addition, comparison of the analgesic affects of Novafen and ibuprofen showed that Novafen had better analgesic effects at 1- and 2-hour intervals postoperatively; however, the differences were not significant.

\section{References}

1. Bamgbose BO, Akinwande JA, Adeyemo WL, Ladeinde AL, Arotiba GT, Ogunlewe MO. Effects of co-administered dexamethasone and diclofenac potassium on pain, swelling and trismus following third molar surgery. Head Face Med. 2005;1(11):1-11.

2. Fisher S, Frame J, Rout $P$, McEntegart D. Factors affecting the onset and severity of pain following the surgical removal of unilateral impacted mandibular third molar teeth. British dental journal. 1988;164(11):351-4.

3. Zuniga JR, Phillips CL, Shugars D, Lyon JA, Peroutka SJ, Swarbrick J, et al. Analgesic safety and efficacy of diclofenac sodium softgels on postoperative third molar extraction pain. Journal of oral and maxillofacial surgery. 2004;62(7):806-15.

4. Peres MFS, Ribeiro FV, Ruiz KGS, Nociti-Jr FH, Sallum EA, Casati MZ. Steroidal and non-steroidal cyclooxygenase-2 inhibitor anti-inflammatory drugs as pre-emptive medication in patients undergoing periodontal surgery. Brazilian dental journal. 2012;23(6):621-8.

5. Seymour R, Ward-Booth P, Kelly P. Evaluation of different doses of soluble ibuprofen and ibuprofen tablets in postoperative dental pain. British Journal of Oral and Maxillofacial Surgery. 1996;34(1):110-4.

6. Merry A, Gibbs R, Edwards J, Ting G, Frampton C, Davies E, et al. Combined acetaminophen and ibuprofen for pain relief after oral surgery in adults: a randomized controlled trial. British journal of anaesthesia. 2010;104(1):80-8. 7. Diamond S, Freitag FG. The use of ibuprofen plus caffeine to treat tension-type headache. Current pain and headache reports. 2001;5(5):472-8.

8. Naseh M, RezaieKalat S. Comparison of the Effects of Celecoxib, Naproxen and Ibuprofen on Pain Control after Periodontal Surgeries. J Mash Dent Sch. 2011;35(4):306-14.

9. Popova C, Mlachkova A, Emilov D. EFFECTIVENESS OF NSAIDs AULIN AND IBU-PROFEN ON THE POSTOPERATIVE PAIN AT GINGIVAL GRAFT PROCEDURES-A PRE-LIMINARY STUDY. J IMAB. 2008;2:12-5.

10. Raisian S, Fallahi HR, Badakhshan L, Zandian D. A randomized double blind controlled trial comparing Ibuprofen versus Ibuprofen plus Acetaminophen plus Caffeine for pain control after impacted third molar surgery. Open Journal of Stomatology. 2012;2012(2):110-5.

11. Haghighat A, Kaviani N, Mosharrafifar M. Evaluation of celecoxib effect in reducing oral ibuprofen after third molar surgery. Journal of Esfehan University of Medical Science. 2007;2(1):29-33.

12. Khan AA, Dionne RA. COX-2 inhibitors for endodontic pain. Endodontic Topics. 2002;3(1):31-40.

13. Rashwan WA. The efficacy of acetaminophen-caffeine compared to Ibuprofen in the control of postoperative pain after periodontal surgery: a crossover pilot study. J Periodontol. 2009;80(6):945-52.

14. Mehrvarzfar P, Abbott $P$, Saghiri M, Delvarani A, Asgar K, Lotfi M, et al. Effects of three oral analgesics on postoperative pain following root canal preparation: a controlled clinical trial. International endodontic journal. 2012;45(1):76-82.

15. Po ALW, Zhang W. Analgesic efficacy of ibuprofen alone and in combination with codeine or caffeine in postsurgical pain: a meta-analysis. European journal of clinical pharmacology. 1998;53(5):303-11. 